\title{
Modeling Approaches Reveal New Regulatory Networks in Aspergillus fumigatus Metabolism
}

\author{
Enzo Acerbi ${ }^{1}$, Marcela Hortova-Kohoutkova ${ }^{2}$, Tsokyi Choera ${ }^{3}$, Nancy Keller ${ }^{3}{ }^{\circledR}$, Jan Fric ${ }^{2,4}(\mathbb{D}$, \\ Fabio Stella ${ }^{5}$, Luigina Romani ${ }^{6}$ and Teresa Zelante ${ }^{6, *}$ \\ 1 Nlytics Pte. Ltd., Singapore 637551, Singapore; contact@nlytics.ai \\ 2 Centre for Translational Medicine, International Clinical Research Centre, St. Anne's University Hospital \\ Brno, 65691 Brno, Czech Republic; marcela.hortova@fnusa.cz (M.H.-K.); jan.fric@fnusa.cz (J.F.) \\ 3 Department of Medical Microbiology and Immunology, Department of Bacteriology, University of \\ Wisconsin, Madison, WI 53706, USA; tchoera@hexagonbio.com (T.C.); npkeller@wisc.edu (N.K.) \\ 4 Institute of Hematology and Blood Transfusion, 12800 Prague, Czech Republic \\ 5 Department of Informatics, Systems and Communication, University of Milano-Bicocca, Viale Sarca 336, \\ Building U14, 20126 Milan, Italy; fabio.stella@unimib.it \\ 6 Department of Experimental Medicine, University of Perugia, 06132 Perugia, Italy; luigina.romani@unipg.it \\ * Correspondence: teresa.zelante@unipg.it; Tel.: +39-075-585-8236
}

Received: 2 June 2020; Accepted: 10 July 2020; Published: 14 July 2020

check for updates

\begin{abstract}
Systems biology approaches are extensively used to model and reverse-engineer gene regulatory networks from experimental data. Indoleamine 2,3-dioxygenases (IDOs)-belonging in the heme dioxygenase family-degrade L-tryptophan to kynurenines. These enzymes are also responsible for the de novo synthesis of nicotinamide adenine dinucleotide (NAD+). As such, they are expressed by a variety of species, including fungi. Interestingly, Aspergillus may degrade L-tryptophan not only via IDO but also via alternative pathways. Deciphering the molecular interactions regulating tryptophan metabolism is particularly critical for novel drug target discovery designed to control pathogen determinants in invasive infections. Using continuous time Bayesian networks over a time-course gene expression dataset, we inferred the global regulatory network controlling L-tryptophan metabolism. The method unravels a possible novel approach to target fungal virulence factors during infection. Furthermore, this study represents the first application of continuous-time Bayesian networks as a gene network reconstruction method in Aspergillus metabolism. The experiment showed that the applied computational approach may improve the understanding of metabolic networks over traditional pathways.
\end{abstract}

Keywords: Aspergillus fumigatus; tryptophan metabolism; modeling; Bayesian networks; continuous time Bayesian networks; gene network reconstruction; gene network inference

\section{Introduction}

Microbial metabolism is under deep investigation because of the recent advances in enabling metagenomic technologies and the urgent need to further understand the functions of microbes able to colonize human tissues. At this stage, information related to different metabolic pathways and advances in metabolomics represent an important tool for drug development and target discovery. Mathematical modeling has been recently applied to predict potential interactions between different pathways in the yeast Saccharomyces cerevisiae [1]. In particular, the production of immunomodulatory metabolites by the fungus Aspergillus fumigatus is of great interest for the impact on the host immune system [2-4]. 
In this contest, the biological functions of several xenobiotic receptors, which are able to recognize microbial metabolites, have been investigated [5]. The Aryl hydrocarbon receptor (AhR), for example, is able to recognize several products of the aminoacidic catabolism, as well as mycotoxins $[5,6]$.

Of interest, in mammalians the activation of $A h R$ in the gut has been proved to monitor anti-inflammatory responses, with protective effects as in candidiasis or in inflammatory bowel disease. In other tissues, such as the pulmonary tract, the role of xenobiotic receptors is very complex and still under debate [7].

The fungus A. fumigatus is able to degrade/utilize the essential amino acid Tryptophan (Trp) into distinct metabolites following three main pathways. One is via the known rate limiting enzyme aromatic aminotransferase (Aro), one via indoleamine 2,3-dioxygenase (Ido) activity, and a third not associated with a specific enzyme but rather several secondary metabolite enzymes that place Trp and prenylated Trp into small non-ribosomally encoded secondary metabolites. Among the aro genes, aroH (Afu2g13630) encodes the putative pyridoxal 5'-phosphate (PLP)-dependent aromatic aminotransferase, which transforms Trp into indolepyruvate [4,8]. Ido genes (idoA Afu3g14250, idoB Afu4g09830, idoC Afu7g02010) encode putative IDOs, which transform Trp to L-kynurenine. The secondary metabolites pathways yield four known toxins, fumiquinazoline, fumitremorgin, fumigaclavine and hexadehydroastechrome [9]. Although the steps of these different metabolite pathways are well-known, the interactions between the two catabolic axes (Ido and Aro) remain unclear. In this study, the generation of high granularity time-course data allowed for a computational analysis of the dynamics of interactions among the genes in these two systems.

The task of uncovering the causal structure (under the following assumptions: causal sufficiency, faithfulness and the causal Markov condition) of regulatory interactions (often referred to as "gene regulatory networks" or GRNs) is a fervent area of research in computational biology [10,11]. A number of approaches have been applied to the GRNs reconstruction problem. Probabilistic graphical models such as Bayesian networks [12] were shown to be powerful tools for solving the GRN reconstruction problem [13], and they led to significant discoveries [14]. When richer time-course measurements started to be made available, Dynamic Bayesian networks (DBNs) gained more and more relevance in the field. Other probabilistic approaches are state space models [15] and probabilistic Boolean networks; [16] however, it has been shown that the latter are outperformed by DBNs for GRN reconstruction problems [17]. Granger causality (GC) is a robust method for analyzing time-course data; since its early introduction, it has been successfully applied to a multitude of domains, such as economics, neuroscience and biology. Continuous-time Bayesian networks (CTBNs) are an emerging approach, which, thanks to their explicit representation of the time, provide state-of-the-art performance for the problem of gene network reconstruction when time-course data are available [18]. Weighted gene co-expression network analysis (WGCNA) is another widely applied methodology. Unlike WGCNA, which is based on pairwise correlation relationships among genes, CTBNs are based on detecting relationships of causality [19] among random variables whose state evolves over time. CTBNs were proven to be a comparable choice to both GC and DBNs for small-scale networks and a preferable choice to both GC and DBNs for networks of large size and when measurements are collected at unevenly spaced time points [18]. A recent review of existing network reconstruction approaches can be found in [20].

Nowadays, the finely grained time-course data generated by high throughput technologies are particularly suitable for computational methodologies which are conceived to exploit the dynamic nature of datasets, like CTBNs. CTBNs have been recently applied to the analysis of molecular data, to investigate the regulatory interactions that characterize pathogenic versus non-pathogenic murine TH17 cells [18] and TH17 cell differentiation in humans, where their application led to the discovery of a new regulator gene [21]. The graphical component of a CTBN provides an intuitive level of abstraction (in the form of a network) of how the regulatory process operates over the duration of the experiment: nodes corresponds to genes, and arcs represent direct probabilistic relationships among 
genes (one gene exerting a direct influence over the other). In this study, the structure of regulatory interactions controlling Trp metabolism was inferred by using CTBNs.

\section{Materials and Methods}

Strains and medium. The strains that were used in this study are listed in Table 1 . The genetic background of the primary strain used in this study was A. fumigatus CEA17 (Table 1). All strains were maintained as glycerol (Panreac, Miami, Florida, USA) stocks, at $-80^{\circ} \mathrm{C}$, and activated on solid glucose minimal media (GMM), at $37^{\circ} \mathrm{C}[22]$.

Table 1. Fungal Strains used in this study.

\begin{tabular}{|c|c|c|c|}
\hline ID & Strain & Genotype & Reference \\
\hline CEA17 & CEA17 pyrG- KU80 & pyrG1, $\Delta a k u B:: p y r G$, pyrG1 & [23] \\
\hline TTC 22.7 & $\triangle a r o H$ & pyrG1, $\triangle a k u$ B::pyrG, pyrG1, $\triangle A F U B \_029280: p y r G$ & This study \\
\hline
\end{tabular}

Genetic manipulations for $A$. fumigatus aroH mutants by protoplasting method. Fungal DNA extraction, gel electrophoresis, restriction enzyme digestion, Southern blotting, hybridization and probe preparation were performed according to standard methods [24]. For DNA isolation, A. fumigatus strains were grown for $24 \mathrm{~h}$ at $37^{\circ} \mathrm{C}$, in static liquid GMM. DNA isolation was performed as described by Sambrook and Russell [24]. Gene deletion mutants in this study were constructed by targeted integration of the deletion cassette through transformation $[25,26]$. The deletion cassettes were constructed by using a double-joint fusion PCR (DJ-PCR) approach $[25,26]$. A. fumigatus protoplast generation and transformation were carried out as previously described [25,26].

Fungal cell culture. Fungal strains were put in culture $\left(1 \times 10^{8}\right.$ conidia/mL $)$ in RPMI 1640 medium (GIBCO, Milano, Italy), for each condition, at $37^{\circ} \mathrm{C}$. Supplemental Trp (Sigma-Aldrich Merck Life Science S.r.l. Milano, Italy) resulted in a final concentration of $100 \mu \mathrm{M}$. Cells were harvested for RNA isolation every $10 \mathrm{~min}$ for $3 \mathrm{~h}$.

RNA isolation and qPCR. Fungal biomass was disrupted by using a FastPrep-FP120 (BIO101) (Qbiogene, Inc, Illkirch, CEDEX, France) at $4.5 \mathrm{~m} / \mathrm{s}$ for $30 \mathrm{~s}$. Samples were left at room temperature for 5 min, in ice, and subsequently centrifuged for $10 \mathrm{~min}$ at $13,000 \mathrm{rpm}$ at $4{ }^{\circ} \mathrm{C}$. Total RNA was extracted from purified cells by the TRIzol method (Invitrogen, Milano, Italy), according to the manufacturer's protocol. The cDNA was synthesized by using the PrimeScript RTreagent kit (TAKARA, Saint-Germain-en-Laye France). Then, qPCR was carried out with primers listed in Table 2, using SybrGreen Expression Master Mix (Thermo Fisher Scientific, Milano, Italy). The qPCR analysis was performed by using a LightCycler II (Roche, Basel, Switzerland). The Ct values of genes of interest were normalized to house-keeping gene $18 \mathrm{~S}(\Delta \mathrm{Ct})$, and the relative expression of each gene of interest was calculated as $2^{-\Delta \mathrm{Ct}}$. All reactions were repeated at least three times, independently, and normalized with $\beta$-actin gene expression.

Table 2. qPCR primers used in this study.

\begin{tabular}{clc}
\hline Gene & \multicolumn{1}{c}{ Primers Sequence $\left(5^{\prime}-3^{\prime}\right)$} & Annealing Temperature $\left({ }^{\circ} \mathbf{C}\right)$ \\
\hline $18 S$ & $\begin{array}{l}\text { Sense } \rightarrow \text { GAGCCGATAGTCCCCCTAAG } \\
\alpha \text { Sense } \rightarrow \text { ATGGCCGTTCTTAGTGGTG } \\
\text { aroH } H\end{array}$ & $\begin{array}{l}\text { Sense } \rightarrow \text { AAAGTCCCGACAGCAATCTACA } \\
\alpha \text { Sense } \rightarrow \text { TGGGACTTTCACGCTAATCTCT }\end{array}$ \\
$i d o A$ & $\begin{array}{l}\text { Sense } \rightarrow \text { ATGCCTGTCTCGCTATGC } \\
\alpha \text { Sense } \rightarrow \text { CTCGGGTGTACGGTTTCG }\end{array}$ & 60 \\
$i d o B$ & $\begin{array}{l}\text { Sense } \rightarrow \text { AGGAAGTTGTCGCTGATTACC } \\
\alpha \text { Sense } \rightarrow \text { ATGCTCGCCGCCATTCTG }\end{array}$ & 55 \\
$i d o C$ & $\begin{array}{l}\text { Sense } \rightarrow \text { TCAGCCAGGATGGCAGTC } \\
\alpha \text { Sense } \rightarrow \text { TCGTCAGTCAGGTCAGGAAG }\end{array}$ & 54 \\
\hline
\end{tabular}


Data preprocessing and learning parameters. Raw data were analyzed by using $\mathrm{R}$ version 3.1.2 and the Bioconductor package. Data were log2 transformed. Four separate networks were learned by using time-course datasets from the experiments wild type, wild type with the addition of Trp, wild type with aroH mutant and wild type with aroH knockout with the addition of tryptophan. Missing values in time-course datasets were replaced with zeroes. The R package ctbn v.1.0 was used for the analysis. Due to the limited amount of data available for each experimental condition, structural learning of continuous-time Bayesian networks (CTBNs) was run multiple times, with varying hyperparameter combinations. Specifically, $\alpha$ was tested for values equal to 3, 2, 1, 0.1, 0.01 and 0.001 , while $\tau$ for values equal to 10, 5, 1, 0.1 and 0.01 . The experimental campaign included testing various discretization approaches, eventually choosing to discretize the data into 3 bins of equal size. This resulted in 30 candidate networks learned for each of the 4 experimental conditions. Only arcs detected in more than $90 \%$ of candidate networks that had at least one arc were considered to be high-confidence and reported in the final graphs shown in Figure 3.

Statistical analysis. Statistical analysis was performed with ANOVA tests and GraphPad Prism 6 software (GraphPad Software, San Diego, CA, USA).

\section{Results and Discussion}

The network shown in Figure 1 represents the known, literature-based Trp catabolic cascade in $A$. fumigatus, which is organized in two separate axes and activated by Trp. One axis is regulated by the ido genes, and a secondary axis is regulated by the aroH gene, in a way that each gene controls the transcription of another gene downstream in the same axis.

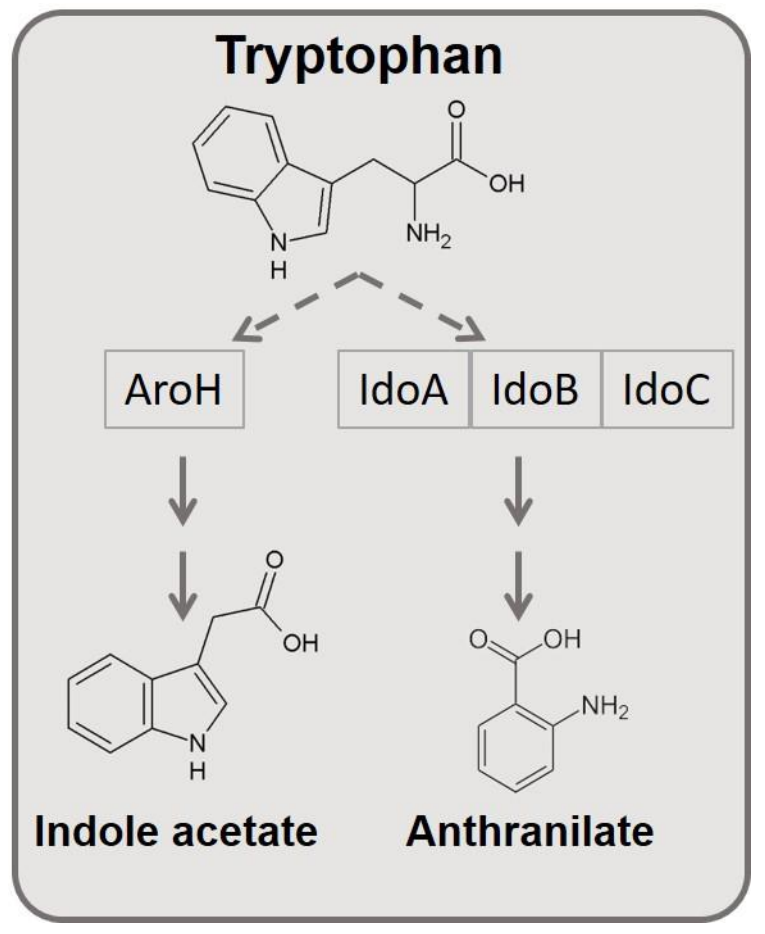

Figure 1. Known literature-based interactions between catabolic axis and the amino acid Trp in A. fumigatus.

The axis regulated by the ido genes leads to kynurenine production. Those metabolites are very well-known in the mammalian metabolism as important modulators of the "Trp starvation" response in inflammation and infection. In particular, L-Kynurenine is known to bind the xenobiotic receptor AhR and reduce T-cell reactivity [27,28]. In addition, when Trp starvation is induced by IDO expression, effector T cells undergo anergy, reducing inflammation. In infections, Trp starvation is also described to reduce microbial metabolism and pathogen virulence [29]. In the fungus Aspergillus, the role of Trp 
metabolism is still under investigation, although several studies already characterized the protein functions of the catabolic cascade [30].

Idos proteins in Aspergillus seem to degrade Trp in to kynurenines, with different affinity for Trp and velocity rate [8]. The Aro pathway in Aspergillus is less characterized, although we have recently characterized the protein function, and we have shown that the catabolic cascade induces the release of indole derivatives as indole acetate from Trp (manuscript submitted) [31].

However, all of these studies highlight the fundamental concept that Trp metabolism can be activated off or on by culturing cells in low-Trp or in high-Trp concentration, respectively.

Therefore, we placed in culture the fungus Aspergillus in conditions of low or high Trp availability, in order to switch off or strongly activate the whole cascade, respectively. The scheme of the experiment and the forward analysis based on expression data are shown in Figure 2. Knowing that both pathways may potentially degrade Trp [4,31], we maximally induced the catabolism on the other axis by removing one axis, as shown by qPCR results (Supplementary Materials Figure S2).

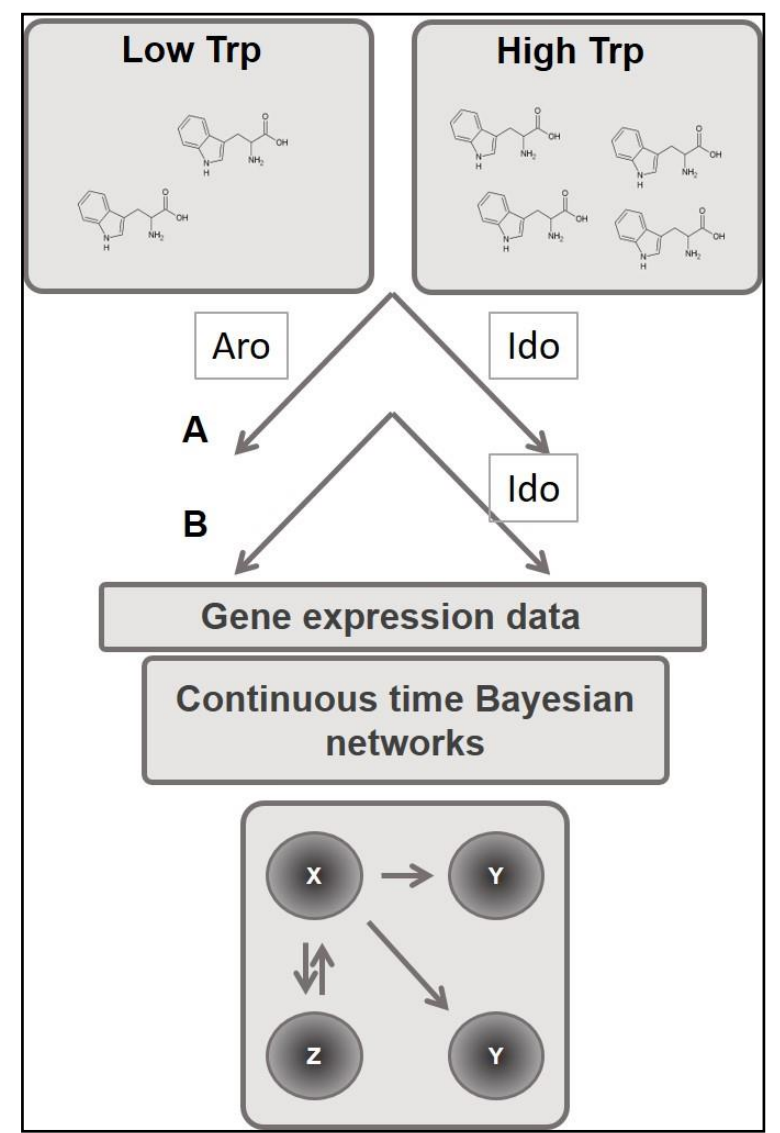

Figure 2. Experimental setting for model construction. A, wild type fungal strain was exposed to different concentrations of Trp (Low or High). B, $\triangle a r o H$ fungal strain exposed to different concentration of Trp (Low or High). X, Y, Z represent generical networks that will be eventually generated in the A and $\mathrm{B}$ conditions.

For this purpose, we used the comparison between two different strains of fungi: the wild-type CEA17 and the mutant strain of Aspergillus for the gene $\operatorname{aroH}$ (see Table 1), obtained by genetic manipulation, using the protoplasting method. The obtained mutants were named in this study TTC22 $\times(\triangle \operatorname{aroH})$.

We selected the removal of the well-characterized rate limiting enzyme Aro for one axis ("condition $\left.\mathrm{B}^{\prime \prime}\right)$ (Figure 2) in comparison with "condition A", where, in the wild-type strain, both axes are active and able to degrade Trp. As mentioned above, for "condition A", we used the wild-type strain CEA17 pyrG- KU80, and for "condition B", we used the mutant strain $\triangle a r o H$. 
In order to analyze the dynamic behavior of the network, we performed experiments by placing cells on low Trp (off experiments) to high Trp (on experiments). We collected samples every $10 \mathrm{~min}$, for up to $3 \mathrm{~h}$. We analyzed expression profiles of catabolic genes by quantitative real-time RT-PCR (q-PCR), focusing on four genes (ido $A, i d o B, i d o C$, and $a r o H$ ). Thus, our network was indeed composed of four different genes, which codify for catabolic enzymes of the Trp cascade.

In the off experiments, Trp availability slightly led to the transcription of all the network genes by inducing two waves of expression for idos and only one wave of expression for $\mathrm{aroH}$. Their dynamic behavior is obvious, as in the on experiments, a seemingly oscillatory behavior is clearly observable for all ido genes but not for aroH (Supplementary Materials Figure S1). Higher peaks of expression are present at 30-40 $\mathrm{min}$ and $160-180 \mathrm{~min}$. The $\mathrm{aroH}$ gene is activated with a different type of kinetic with only picks at $150 \mathrm{~min}$ (Supplementary Materials Figure S1).

Compared to "condition A" (Supplementary Materials Figure S1), in "condition B" (Supplementary Materials Figure S2), aroH deletion led to an increase of $i d o B$ and idoC expression for both the on and the off experiments. These results show that the two pathways catalyzed by Ido and AroH are probably reciprocally regulated. Indeed, the deletion of $a r o H$ leads to higher expression of $i d o$ genes. This was clearer for $i d o B$ and $i d o C$ compared to $i d o A$. Moreover, the deletion of $i d o s$ increased aroH expression. Although this was expected, it is still unclear what the reason for it and what the physiological role of the activation of the two alternative pathways are. Based on the different impacts of metabolites on host immune system, the release of different metabolites (kynurenines or indoles) is extremely of interest.

The model successfully predicted the systems behavior during the on experiment: In "condition $\mathrm{A}^{\prime \prime}$, all genes in the network are inferred as being independently activated in response to Trp (Figure 3A and Supplementary Materials Figure S1). This suggests that, in the condition with a higher availability of Trp, the different enzymes are independently activated in degrading Trp. In aro deficiency ("condition $\left.\mathrm{B}^{\prime \prime}\right)$, the model detects interactions between $i d o A$ and $i d o C$ (Figure 3B). This relationship underlines a determinant action of $i d o A$ and its derived metabolites in the activation of other genes in the network. This also emerges in the off experiments, where $i d o A$ is detected as primarily regulating the whole network (Figure 3B).

In addition, the CTBNs-based model also suggests that, in condition of aro deficiency, an increased interaction between ido genes and increased production of Ido-derived metabolites is possible.

Modeling has become recently a significant tool to understand microbial ecosystem in different contexts, such as clinical microbiology, food fermentation and microbial metabolism. Importantly, modeling may be used to better understand the co-metabolism between the host and pathogens in the context of the immune response [32].

These results also underline the aspect that models are simulated laboratories where it can be artificially improved the experimental design. Future studies, in this particular field, may enhance our understanding on host-pathogen interactions in conditions of nutrient availability. Studies in the field of host-pathogen interactions based on Bayesian networks have been already presented [33]. Those models analyzed the impact of climatic variables or the activation of the immune system during fungal infections in amphibians. Pivotally, in the future, modeling may be used to understand how co-metabolism may affect the outcome of the infection, together with other validated variables.

In particular, in the context of the model presented, the use of additional Aspergillus mutants for Trp biosynthesis and catabolism may increase the complexity of the model design.

Based on the result of our study, more comprehensive models based on CTBNs, including, for instance, a detailed construction of Trp metabolism, or based on different experimental conditions, can be developed in the future. This study confirms CTBNs to be an effective methodology for computational gene network reconstruction, and a useful hypothesis generation tool in the study of Trp metabolism: CTBNs could aid in the discovery of novel interactions, supporting the elucidation of those biological processes that strongly impact the fungal fitness, as well as the human immune system during infection. 
A
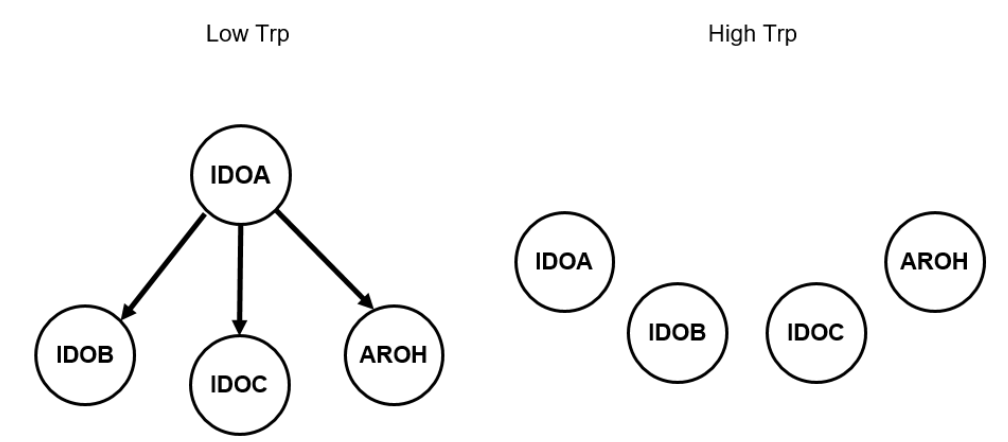

B

Low Trp

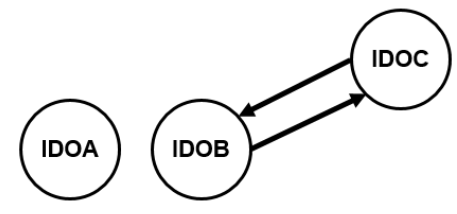

Figure 3. Networks of predicted regulatory interactions learned from RT-PCR time-course data for wild-type strain (Panel A), and aroH deletion (Panel B) experimental conditions. In both cases, separate networks were inferred by using data from low-Trp (off) and high-Trp (on) experiments, for a total of four separate networks.

Supplementary Materials: The following are available online at http://www.mdpi.com/2309-608X/6/3/108/s1. Figure S1: qPCR expression data of wild-type A. fumigatus strain. Figure S2: qPCR expression data of $\triangle a r o H$ A. fumigatus strain. The $\mathrm{R}$ script and the data used in this study are available at the following URL: https: //gitlab.com/enzoace/modellingaspergillus.

Author Contributions: Conceptualization, T.Z., E.A., F.S., N.K. and L.R.; investigation, E.A., T.Z. and J.F.; methodology, T.C., M.H.-K. and T.Z.; resources, T.Z., J.F. and N.K.; project administration, T.Z. and J.F.; and funding acquisition, T.Z. and J.F. All authors have read and agreed to the published version of the manuscript.

Funding: The research was supported by European Social Fund and European Regional Development Fund-Project MAGNET (No. CZ.02.1.01/0.0/0.0/15_003/0000492) and ENOCH (CZ.02.1.01/0.0/0.0/16_019/0000868), and by Ministry of Health of the Czech Republic, grant nr. NV18-06-00529. The Italian Grant "Programma per Giovani Ricercatori-Rita Levi Montalcini 2013" (Project number: PGR13XNIDJ) to T.Z.

Conflicts of Interest: The authors declare no conflict of interest.

\section{References}

1. Cantone, I.; Marucci, L.; Iorio, F.; Ricci, M.A.; Belcastro, V.; Bansal, M.; Santini, S.; di Bernardo, M.; di Bernardo, D.; Cosma, M.P. A yeast synthetic network for in vivo assessment of reverse-engineering and modeling approaches. Cell 2009, 137, 172-181. [CrossRef] [PubMed] 
2. Choera, T.; Zelante, T.; Romani, L.; Keller, N.P. A Multifaceted Role of Tryptophan Metabolism and Indoleamine 2,3-Dioxygenase Activity in Aspergillus fumigatus-Host Interactions. Front. Immunol. 2017, 8 , 1996. [CrossRef] [PubMed]

3. Keller, N.P. Fungal secondary metabolism: Regulation, function and drug discovery. Nat. Rev. Microbiol. 2019, 17, 167-180. [CrossRef]

4. Wang, P.M.; Choera, T.; Wiemann, P.; Pisithkul, T.; Amador-Noguez, D.; Keller, N.P. TrpE feedback mutants reveal roadblocks and conduits toward increasing secondary metabolism in Aspergillus fumigatus. Fungal Genet. Biol. 2016, 89, 102-113. [CrossRef] [PubMed]

5. Arenas-Huertero, F.; Zaragoza-Ojeda, M.; Sanchez-Alarcon, J.; Milic, M.; Segvic Klaric, M.; Montiel-Gonzalez, J.M.; Valencia-Quintana, R. Involvement of Ahr Pathway in Toxicity of Aflatoxins and Other Mycotoxins. Front. Microbiol. 2019, 10, 2347. [CrossRef]

6. Ayed-Boussema, I.; Pascussi, J.M.; Zaied, C.; Maurel, P.; Bacha, H.; Hassen, W. Ochratoxin A induces CYP3A4, 2B6, 3A5, 2C9, 1A1, and CYP1A2 gene expression in primary cultured human hepatocytes: A possible activation of nuclear receptors. Drug. Chem. Toxicol. 2012, 35, 71-80. [CrossRef] [PubMed]

7. Neavin, D.R.; Liu, D.; Ray, B.; Weinshilboum, R.M. The Role of the Aryl Hydrocarbon Receptor (AHR) in Immune and Inflammatory Diseases. Int. J. Mol. Sci. 2018, 19, 3851. [CrossRef] [PubMed]

8. Yuasa, H.J.; Ball, H.J. The evolution of three types of indoleamine 2,3 dioxygenases in fungi with distinct molecular and biochemical characteristics. Gene 2012, 504, 64-74. [CrossRef] [PubMed]

9. Raffa, N.; Keller, N.P. A call to arms: Mustering secondary metabolites for success and survival of an opportunistic pathogen. PLoS Pathog. 2019, 15, e1007606. [CrossRef]

10. Marbach, D.; Costello, J.C.; Kuffner, R.; Vega, N.M.; Prill, R.J.; Camacho, D.M.; Allison, K.R.; Consortium, D.; Kellis, M.; Collins, J.J.; et al. Wisdom of crowds for robust gene network inference. Nat. Methods 2012, 9, 796-804. [CrossRef]

11. Feist, A.M.; Herrgard, M.J.; Thiele, I.; Reed, J.L.; Palsson, B.O. Reconstruction of biochemical networks in microorganisms. Nat. Rev. Microbiol. 2009, 7, 129-143. [CrossRef] [PubMed]

12. Friedman, N.; Linial, M.; Nachman, I.; Pe'er, D. Using Bayesian networks to analyze expression data. J. Comput. Biol. 2000, 7, 601-620. [CrossRef] [PubMed]

13. Zhu, J.; Wiener, M.C.; Zhang, C.; Fridman, A.; Minch, E.; Lum, P.Y.; Sachs, J.R.; Schadt, E.E. Increasing the power to detect causal associations by combining genotypic and expression data in segregating populations. PLoS Comput. Biol. 2007, 3, e69. [CrossRef] [PubMed]

14. Sachs, K.; Perez, O.; Pe'er, D.; Lauffenburger, D.A.; Nolan, G.P. Causal protein-signaling networks derived from multiparameter single-cell data. Science 2005, 308, 523-529. [CrossRef] [PubMed]

15. Hirose, O.; Yoshida, R.; Imoto, S.; Yamaguchi, R.; Higuchi, T.; Charnock-Jones, D.S.; Print, C.; Miyano, S. Statistical inference of transcriptional module-based gene networks from time course gene expression profiles by using state space models. Bioinformatics 2008, 24, 932-942. [CrossRef]

16. Shmulevich, I.; Dougherty, E.R.; Kim, S.; Zhang, W. Probabilistic Boolean Networks: A rule-based uncertainty model for gene regulatory networks. Bioinformatics 2002, 18, 261-274. [CrossRef]

17. Li, P.; Zhang, C.; Perkins, E.J.; Gong, P.; Deng, Y. Comparison of probabilistic Boolean network and dynamic Bayesian network approaches for inferring gene regulatory networks. BMC Bioinform. 2007, 8 (Suppl. 7), S13. [CrossRef]

18. Acerbi, E.; Zelante, T.; Narang, V.; Stella, F. Gene network inference using continuous time Bayesian networks: A comparative study and application to Th17 cell differentiation. BMC Bioinform. 2014, 15, 387. [CrossRef]

19. Pearl, J. An introduction to causal inference. Int. J. Biostat. 2010, 6. [CrossRef]

20. Delgado, F.M.; Gomez-Vela, F. Computational methods for Gene Regulatory Networks reconstruction and analysis: A review. Artif. Intell. Med. 2019, 95, 133-145. [CrossRef]

21. Acerbi, E.; Vigano, E.; Poidinger, M.; Mortellaro, A.; Zelante, T.; Stella, F. Continuous time Bayesian networks identify Prdm1 as a negative regulator of TH17 cell differentiation in humans. Sci. Rep. 2016, 6, 23128. [CrossRef] [PubMed]

22. Shimizu, K.; Keller, N.P. Genetic involvement of a cAMP-dependent protein kinase in a G protein signaling pathway regulating morphological and chemical transitions in Aspergillus nidulans. Genetics 2001, 157, 591-600. 
23. da Silva Ferreira, M.E.; Kress, M.R.; Savoldi, M.; Goldman, M.H.; Hartl, A.; Heinekamp, T.; Brakhage, A.A.; Goldman, G.H. The akuB(KU80) mutant deficient for nonhomologous end joining is a powerful tool for analyzing pathogenicity in Aspergillus fumigatus. Eukaryot. Cell 2006, 5, 207-211. [CrossRef]

24. Sambrook, J.; Russell, D. Molecular Cloning: A Laboratory Manual; Cold Spring Harbor Laboratory Press: New York, NY, USA, 2001.

25. Szewczyk, E.; Nayak, T.; Oakley, C.E.; Edgerton, H.; Xiong, Y.; Taheri-Talesh, N.; Osmani, S.A.; Oakley, B.R. Fusion PCR and gene targeting in Aspergillus nidulans. Nat. Protoc. 2006, 1, 3111-3120. [CrossRef] [PubMed]

26. Lim, F.Y.; Sanchez, J.F.; Wang, C.C.; Keller, N.P. Toward awakening cryptic secondary metabolite gene clusters in filamentous fungi. Methods Enzymol. 2012, 517, 303-324. [PubMed]

27. Lee, G.K.; Park, H.J.; Macleod, M.; Chandler, P.; Munn, D.H.; Mellor, A.L. Tryptophan deprivation sensitizes activated $\mathrm{T}$ cells to apoptosis prior to cell division. Immunology 2002, 107, 452-460. [CrossRef]

28. Bessede, A.; Gargaro, M.; Pallotta, M.T.; Matino, D.; Servillo, G.; Brunacci, C.; Bicciato, S.; Mazza, E.M.; Macchiarulo, A.; Vacca, C.; et al. Aryl hydrocarbon receptor control of a disease tolerance defence pathway. Nature 2014, 511, 184-190. [CrossRef]

29. Badawy, A.A. Kynurenine Pathway of Tryptophan Metabolism: Regulatory and Functional Aspects. Int. J. Tryptophan Res. 2017, 10, 1178646917691938. [CrossRef]

30. Ball, H.J.; Jusof, F.F.; Bakmiwewa, S.M.; Hunt, N.H.; Yuasa, H.J. Tryptophan-catabolizing enzymes-Party of three. Front. Immunol. 2014, 5, 485. [CrossRef]

31. Dindo, M.; Costanzi, E.; Pieroni, M.; Costantini, C.; Annunziato, G.; Bruno, A.; Keller, N.P.; Romani, L.; Zelante, T.; Cellini, B. Biochemical Characterization of Aspergillus fumigatus AroH, a Putative Aromatic Amino Acid Aminotransferase. Front. Mol. Biosci. 2018, 5, 104. [CrossRef]

32. Esser, D.S.; Leveau, J.H.; Meyer, K.M. Modeling microbial growth and dynamics. Appl. Microbiol. Biotechnol. 2015, 99, 8831-8846. [CrossRef] [PubMed]

33. Bower, D.S.; Mengersen, K.; Alford, R.A.; Schwarzkopf, L. Using a Bayesian network to clarify areas requiring research in a host-pathogen system. Conserv. Biol. 2017, 31, 1373-1382. [CrossRef] [PubMed]

(C) 2020 by the authors. Licensee MDPI, Basel, Switzerland. This article is an open access article distributed under the terms and conditions of the Creative Commons Attribution (CC BY) license (http://creativecommons.org/licenses/by/4.0/). 